CULTURA, LENGUAJE Y REPRESENTACIÓN / CULTURE, LANGUAGE AND REPRESENTATION • ISSN 1697-7750 • VOL. XV \2016, PP. 119-137 REVISTA DE ESTUDIOS CULTURALES DE LA UNIVERSITAT JAUME I / CULTURAL STUDIES JOURNAL OF UNIVERSITAT JAUME I

DOI: HTTP://DX.DOI.ORG/10.6035/CLR.2016.15.8

\title{
Del cuiloni al homosexual: sexualidades masculinas disidentes en El Salvador entre 1932-1992
}

\author{
From cuiloni to the homosexual: Male dissident sexualities \\ in El Salvador between 1932-1992
}

\author{
AMARAL PALEVI Gómez ARÉVALO \\ UNIVERSIDAD FEDERAL PERNAMBUCO
}

Recibido: 11-10-2014

Aceptado: 05-03-2015

RESUMEN: Este artículo tiene el objetivo de visibilizar la existencia de sexualidades masculinas disidentes y su tratamiento en el marco del orden sexo-género binario dominante entre 1932 y 1992 en El Salvador. La metodología utilizada fue la exploratoria, integrando la revisión bibliográfica y entrevistas para recuperar información de sexualidades masculinas disidentes a partir del genocidio indígena de 1932 hasta la culminación de la guerra interna en 1992. Los discursos y prácticas de la sexualidad analizados durante el periodo de estudio reflejan un patrón del ejercicio de la homofobia que conduce desde el menosprecio, la marginación, la exclusión o la tortura hasta la desaparición y asesinato de aquellos que se encuentran en las fronteras o atravesaron las barreras del binarismo sexual hegemónico.

Palabras clave: El Salvador, cuerpo, sexualidad, diversidad sexual, homofobia.

ABSTRACT: This paper aims to make visible the male dissident sexualities in the period between 1932 and 1992 in El Salvador, and how the sex-gender binary order affect them. We use an exploratory methodology, integrating a literature review and interviews with activists to retrieve information from male sexuality dissident indigenous genocide in from 1932 until the beginning of the internal war in 1992. Over sixty years on discourses and practices of dissident sexuality, we have found a pattern of homophobia that leads to marginalization, exclusion, torture up the disappearance and murder of those who are in the borders or who crossed the barriers of the hegemonic sexual binarism.

Keywords: El Salvador, body, sexuality, sexual diversity, homophobia. 


\section{Introducción}

Adentrarse en temas de sexualidad en El Salvador es un desafío que pocos autores se han atrevido a realizar debido, principalmente, a la connotación de tabú que se ha designado por parte del pensamiento conservador imperante en el país, que ha tratado de ocultar el cuerpo, el género y la sexualidad de los discursos públicos, académicos y oficiales, dando como resultado una reducida producción académica existente.

El Salvador ha sido mundialmente conocido por los procesos de violencia que al interior de su territorio se han gestado. Para su transformación se recurrió a pactos y acuerdos para modificar la estructura donde se reproduce la violencia; en este sentido cabe recordar los Acuerdos de Paz de 1992, que pusieron fin a 12 años de guerra interna que dio como saldo la muerte de 75.000 personas (Organización de las Naciones Unidas, 1993). El efecto de estos acuerdos fue restringido, ya que si bien es cierto que se detuvo la violencia armada militar, la violencia social solamente se recicló. Lo anterior se comprueba en las cifras de personas fallecidas por actos de violencia, que alcanzaron en 2011 un total de 4.371 (Organización Mundial de la Salud, 2014: 13), cifra muy próxima, por ejemplo, a las 5.962 víctimas de la población civil en el año 1982 como consecuencia de la guerra (Organización de las Naciones Unidas, 1993: 18). Todo ello nos plantea la necesidad de procurar nuevos caminos para analizar las relaciones de poder entre hombres y mujeres y entre los mismos hombres en este contexto específico.

En este sentido, este artículo pretende visibilizar la existencia de sexualidades masculinas disidentes al orden binario hegemónico y profundizar en el tratamiento que recibieron entre 1932 y 1992. Este aporte académico es resultado parcial de mi línea de investigación sobre estudios LGBT, orientada a la comprensión del sujeto salvadoreño desde su cuerpo y sexualidad. La metodología utilizada es exploratoria. Para este fin, y tomando en cuenta que investigar sobre la historia en El Salvador es recurrir a «fuentes inéditas» (Prudhmme, 2010), y más aún cuando se investiga sobre cuerpo, género y sexualidad, y en particular sobre sexualidades masculinas disidentes, se recurre a la literatura salvadoreña como fuente principal de información, siendo un aspecto coherente con las palabras de Foucault (1972) cuando señala que realizamos un «trabajo arqueológico». También se utilizaron publicaciones nacionales e internacionales que contienen aportes sobre el objeto de estudio o plantean categorías para su análisis. Por último, se realizó una entrevista a Joaquín Cáceres, coordinador de proyectos y miembro fundador de la asociación salvadoreña de derechos humanos Entre Amigos y se revisaron documentos 
que integran el Archivo Histórico de la Diversidad Sexual recopilados por la organización Acción por la Memoria y Apoyo al Trabajo para la Equidad en El Salvador (www.amate.org.sv).

Este artículo se organiza a partir de la relectura básica de cuatro periodos históricos de El Salvador. El primer apartado expondrá la estructura de la jerarquización de la sexualidad de la cosmovisión indígena por medio de un relato oral de origen náhuat-pipil ${ }^{1}$ y un breve análisis de las obras de Salvador Salazar Arrúe, conocido como Salarrué. En el segundo apartado se abordará el uso del travestismo como medio de ataque político en la época de represión política previa a la guerra interna. En el tercero se profundiza en la creación de un espacio de sociabilidad para sexualidades masculinas disidentes en la década de 1970. En el cuarto apartado nos preguntamos: ¿la guerra interna de El Salvador en la década de 1980 fue hecha por solo heterosexuales? Tras el análisis, podemos afirmar que durante los sesenta años de historia de las sexualidades masculinas disidentes existe un patrón de homofobia que conduce al menosprecio, la marginación, la exclusión, la tortura, el desaparecimiento y el asesinato de todo aquel que está y atraviesa las fronteras del binarismo sexual hegemónico.

\section{La jerarquía de lo sexual}

La historia contemporánea de El Salvador está marcada por la violencia en sus más diversas formas. Para tener un referente, en 1932 se realizó el etnocidio del último reducto de importancia del pueblo pipil, conocido como los izalcos, en la zona occidental de El Salvador. Con un saldo histórico de 30.000 muertos con la subsecuente fragmentación cultural, social y política (Audiovisuales UCA, 2007). Con esta masacre se inauguran las dictaduras militares que gobernarán por más de 50 años.

Antes de la realización de este etnocidio, el alemán Leonhard SchultzeJena transcribió en 1930 más de 50 relatos de mitos en lengua náhuat-pipil. Entre sus relatos existe uno que habla sobre la sexualidad, específicamente sobre el acto sexual entre dos hombres. Este relato se llama «La boda del vagabundo» (Lara-Martínez, 2012: 84-86), donde se presenta un acto de penetración sexual al cuerpo de un hombre mayor por parte de otro más joven que

1. Los pipiles son parte de la macrolengua yuto-nahua, relacionados a la cultura tolteca clásica. Los pipiles era el grupo cultural de mayor relevancia a la llegada de los españoles en el actual territorio de El Salvador, que en ese momento se le conocía por el nombre de cushcatan. 
le le había ganado una apuesta para casarse con su hija y poseerlo antes de la boda con ella. Esto en ningún momento se puede catalogar como homosexual, dado que la orientación sexual no es una condición para la realización de dicho acto sexual.

Así, este acto queda integrado a una práctica sexual puntual del ejercicio del poder de un hombre sobre otro, lo que Bourdieu denomina como libido dominandi (Bourdieu, 1999: 31), en su mayor expresión de poder masculino entre los hombres: la penetración sexual. Los pipiles solamente reconocían a la persona que ejercía el rol receptivo o pasivo designado como cuiloni (palo que hecha flores) y el tecuilonti como el que ejerce el rol activo, con una clara connotación de jerarquía social de dominador-dominado (Lara-Martínez, 2012: 230-231), sin ninguna connotación a una orientación sexual específica.

Con la implementación de las dictaduras militares, los diversos ámbitos de la vida salvadoreña son impregnados por el temor del ejercicio de la violencia homicida, al ser esta institucionalizada. La cultura de dominación se fundamentó en ser machista, mestizo-criolla, blanca, hispanohablante, católica conservadora y heterosexista-normativa. En este ámbito, el psicólogo social Martín-Baró (2012: 164-169) manifiesta que la socialización sexual de los roles de género entre hombres y mujeres se constituye como esencialista. De esta manera, se permite una subordinación social de las diferencias sexuales que sirve para perpetuar los intereses de los opresores en lo político, al sometimiento de la mujer al hombre en lo social y a la heterosexualidad como la única forma aceptada para expresar la sexualidad en relación a la cultura.

Las relaciones de poder que se ejercen desde la sexualidad es un tema poco abordado en El Salvador. No obstante, el antropólogo lingüista Lara-Martínez (2012: 180), lo coloca como uno de los principios de la construcción de la identidad sexual y las relaciones de poder entre los hombres; esta conclusión la obtiene al analizar las obras emblemáticas de la cultura salvadoreña de Cuentos de Barro (1933) y Cuentos de cipotes $^{2}$ (1945/1961) escritas por Salarrué.

El Salvador concede a Salvador Salazar Arrúe la categoría de icono de la literatura costumbrista salvadoreña. Nacido en Sonsonate, lugar de asentamiento de los izalcos, permite que su literatura se nutra de la cosmovisión indígena. En esta literatura, «raptos y venganzas de amor, velorios y duelos a machete, sacas de aguardiente clandestino y embrujos, procesiones de rogativa para la lluvia» (Ramírez, 1985: XVI), forman parte del imaginario contextual

2. Cipote es una forma coloquial de designar a un niño o niña, y en este caso su uso en plural hace referencia a la niñez salvadoreña. No obstante, en el contexto ibérico, haciendo uso de un lenguaje vulgar, este mismo concepto hace referencia al miembro viril. 
salvadoreño, que es retratado por Salarrué, incluyendo las formas de expresión de la sexualidad.

Cuando ingresamos al área de la sexualidad en las obras de este autor identificamos las siguientes características sexuales: $a$ ) la intimidad de dos cuerpos sexuados que se acercan y se palpan se califica como algo sucio; $b$ ) la violencia rige lo sexual; $c$ ) la sexualidad como ejercicio de poder (Lara-Martínez, 2012: 171-181). Respecto a la manifestación de sexualidades disidentes en Cuentos de cipotes, aunque no se manifiesta directamente un personaje homosexual, existe un alto grado de sublimación del erotismo anal. En toda la obra de Salarrué queda expuesta con diferentes nominaciones la importancia del tabú anal en la construcción de la identidad masculina hegemónica, ya que «[...] antes que la mujer, el culero define la verdadera identidad masculina en la mente del cipote salvadoreño popular» (Lara-Martínez, 2012: 180). En tal sentido, el heterosexismo patriarcal desplaza este tabú a lo ininteligible, a lo marginal y lo privado, promoviendo que la heterosexualidad sea la única relación socialmente aceptada, respetada y legalizada. Para cualquiera que ose atravesar y estar en los márgenes de esa sexualidad permitida, se expone a dejar la categoría de ser humano y pasaría a un estado donde el «culero representa el oprimido por antonomasia» (Lara-Martínez, 2012: 180).

El uso de la violencia hacia hombres que escapan o están en las fronteras de la heterosexualidad, es una forma de construir y reafirmar la identidad masculina hegemónica (Barrientos, 2015: 129) como sistema político de opresión que al mismo tiempo defiende, afirma y perpetua la heterosexualidad como norma y guion de vida para hombres y mujeres.

\section{Travestismo y represión política}

Después de los sucesos de 1932 las dictaduras militares se instauran en El Salvador. Cada dictador militar asume el rol de patriarca y difunde una imagen ejemplificadora para hombres y mujeres por su papel de garante del orden, donde no duda en aplicar la violencia en los pobladores del país si la situación lo amerita.

En este régimen, donde la libertad de expresión es limitada, la disidencia política procura formas culturales para expresar sus inconformidades sin llegar a ser tan explícitos en sus afrentas contra los detenedores del poder. Es así que el travestismo político adquiere notoriedad. Pero para hablar de ese travestismo es necesario que detallemos algunas nociones del travestismo cultural en El Salvador. 
El travestismo cultural es una práctica común que describiremos por medio de Los viejos de agosto, siendo esta una representación bailada compuesta por personas que se disfrazan de diferentes personajes de la mitología de El Salvador durante las fiestas patronales de la capital, que se celebran cada año del 1 al 6 de agosto.

Entre los personajes más populares se encuentran el Cipitío, la Siguanaba, el diablito, los chapetones y varias representaciones de travestismo exagerado, prioritariamente hombres representando mujeres. En este caso particular deseo hacer una referencia al caso de la Siguanaba. En la mitología cuscatleca, ${ }^{3}$ que no está exenta de ser misógina, nos narra la historia de una mujer indígena hermosa que hace uso de un embrujo para casarse con el hijo del dios de la lluvia, Tlaloc. Esta mujer, cuando su esposo se encontraba fuera de su hogar, tenía encuentros sexuales con otros hombres y descuidaba la atención de su hijo: el Cipitío.

Ella forjó un plan para matar a su esposo y colocar su corona en la cabeza de uno de sus amantes. Todo este plan fue descubierto por Tlaloc, que la maldijo a ser fea, con senos que llegaban hasta sus rodillas, con uñas que nunca podrían ser cortadas, con un cabello largo y desordenado, la cual deberá deambular por la eternidad para purgar sus errores. Aparentando un rostro y cuerpos bello a los hombres que la encontraran solitaria en caminos o en los cauces de los ríos, en un primer momento, para luego mostrar su horrenda forma y burlarse de ellos. O como la define Salarrué: «Sihuanahual (La Sigua), la de dos caras, que enamora y luego espanta» (Salazar Arrué, 2010: 180).

Cuando la Siguanaba es representada en los desfiles de las fiestas patronales, siempre es un hombre que realiza ese rol. Por un lado, se vuelve un recordatorio para el inconsciente heterosexual (Wittig, 2010: 55), donde las mujeres deben subyugar su sexualidad en el dominio de un solo hombre para no padecer los castigos a los que la Sigua está condenada para la eternidad. En estas representaciones, la exageración de los atributos físicos que realizan los hombres a estos personajes, las bromas que llevan a cabo o el simple atuendo que utilizan marca una agenda cultural y política de reproducción de la masculinidad hegemónica. De esta manera, se crea una vigilancia de la sexualidad en donde, quien desee ser efectivamente un hombre, nunca debe ser representado como un personaje travestido, ya que si esto acontece será simbólicamente llevado al campo de lo débil, de lo no valorado, de lo castigado, de lo reprimido, de lo cohibido, de lo preso o, en otras palabras, de lo femenino.

3. Cuscatleco/a es un gentilicio para denominar a los salvadoreños/as. Este deriva de Cuscatlán. 
Con la implementación de las dictaduras militares por hombres y la promoción de una cultura de la dominación machista y heterosexista-normativa, la izquierda política de El Salvador utiliza el travestismo político para denunciar las arbitrariedades de los militares y la oligarquía por medio de la «humillación del otro por su afeminamiento» (Lara-Martínez, 2012: 12), que se realizaba en los célebres desfiles bufos de los estudiantes de la Universidad de El Salvador. No obstante, dichas representaciones de género en vez de colocar en discusión la orientación sexual, se convierten en un dispositivo de control y protección de las fronteras de la heterosexualidad que denigran todas aquellas identidades sexuales subversivas que generan pánico en el heterosexismo normativo, al comprender que en su interior cohabitan posibilidades lésbicas, gays, bisexuales y trans (Butler, 2005: 185; 2007: 186), incluso en la izquierda política, la cual, como veremos en un siguiente apartado, reproduce la homofobia en sus prácticas institucionales.

En este contexto de polaridad entre la izquierda política y la dictadura militar, las sexualidades masculinas disidentes no tuvieron ninguna posibilidad de hacer su manifestación en la escena social o política. La lucha contra el comunismo por el lado militar y la supervivencia e intento de modificar el Estado por parte de la izquierda monopolizaron los discursos, creando un metarelato hegemónico que impregnaba la vida cotidiana de todos los salvadoreños: la lucha por el poder político. La sexualidad en su más amplio sentido, y la homosexualidad en un sentido específico, fueron remitidas al silencio político, lo cual contribuyó a la perpetuación del heterosexismo.

Los hombres con prácticas sexuales disidentes que existieron en este período se posicionaron muy probablemente en uno u otro bando, pero sus prácticas sexuales se mantuvieron en la marginalidad social. Respecto a este punto, al hablar de marginalidad social me estoy refiriendo a los espacios de expresión de su sexualidad, confinada a lugares que transitan desde lo marginal a lo excluido. Muestra de estos espacios es el mítico salón-bar La Praviana en el centro histórico de San Salvador. ${ }^{4}$ Este era reconocido como punto de encuentro de travestis, siendo esta la forma de designar a las personas que presentaban una orientación sexual diferente a la norma heterosexual. Muchos de ellos eran pobres que ejercían la prostitución como medio de vida, todo ello a pesar de los riesgos a ser víctimas de la violencia homofóbica que otros pudieran ejercer sobre ellos.

4. Información proporcionada por Joaquín Cáceres en una entrevista realizada el 20 de agosto de 2014. 


\section{Oráculos: un espacio de sociabilidad}

En la década de 1970, El Salvador vive la época de la represión política, donde se implementa la doctrina de seguridad nacional, la cual define como enemigos internos a movimientos sociales organizados de campesinos, estudiantes, sindicatos laborales y profesores. Estos colectivos estaban compuestos mayoritariamente por hombres, estaban inspirados en la victoria de la revolución cubana y realizaron un proceso de organización político-social nunca más visto en la historia reciente del país (Martínez, 2004).

Las doctrinas de seguridad interna y la organización de los movimientos sociales, como era de esperar, se confrontan, y este choque da como resultado muertes y desapariciones políticas. A pesar de estas actuaciones de exterminio, la resistencia política por parte de las organizaciones de izquierda no decayó, sino al contrario: fue generando simpatía popular. A nivel de la sociedad civil se desarrollaron estrategias para evadir a los cuerpos de seguridad y los escuadrones de la muerte. ${ }^{5}$

Respecto a las sexualidades masculinas disidentes, aparece una referencia clara a las normas homofóbicas que condicionan su existencia por medio de la obra artística San Sebastián (Ernesto San Avilés, 1974), la cual tiene vigencia a día de hoy. Este cuadro está compuesto por un marco que representa una ventana que expone en primer plano una espalda y unos glúteos que se supone pertenecen al cuerpo de un hombre diseñado bajo el canon greco-latino de la figura masculina, con una flecha insertada en uno de los glúteos. En vez de sangre, de la herida se desprende una gota de líquido transparente que alude al semen (Grégori, 2007); a la base de la ventana-marco de la pintura, una fruta y al extremo derecho, casi imperceptible, una mosca.

Al hacer un análisis de la obra, podemos señalar que, al no mostrar el rostro del personaje principal del cuadro, nos da referencia del campo de lo abyecto al cual están sancionados aquellos que están vivos pero que son distintos a los vivos (Butler, 2010), por ello su dolor, agonía y sufrimiento no se percibe, ya que es una vida precaria (Butler, 2010: 33), de un cuerpo que no importa por no

5. Los escuadrones de la muerte eran grupos organizados clandestinos con conexiones a los cuerpos represivos del Estado que se dedicaban a realizar acciones paralelas o encubiertas contra la población organizada y civil. La estrategia de terror de los escuadrones de la muerte para disuadir a la población en el involucramiento de las organizaciones sociales era la realización de procesos selectivos de eliminación de personas y la exposición de sus cadáveres con muestras de tortura en vías públicas de constante tráfico, como la carretera Panamericana, que atraviesa el país de occidente a oriente, y/o en espacios naturales, como la zona de lava petrificada del volcán de San Salvador, conocida como el Playón. 
cumplir la función reproductora, que llegó a constituirse como tal al traspasar los tabúes sexuales - la fruta- de la heterosexualidad obligatoria (Butler, 2007: 36). El sentir placer y goce sexual a través del uso del ano -los glúteos-, en donde las prácticas sexuales -el semen- son actos de disfrute sexual, es algo que condena a la muerte, física o social, de sus practicantes -la flecha- que magistralmente está insertada en el glúteo derecho, la cual fue lanzada por los que intentan prohibir las sexualidades disidentes con el ejercicio del poder que ostentan. La mosca es el corolario de la matriz general de la sexualidad en El Salvador -marco-ventana-, la cual es catalogada como sucia y, por tal razón, la base de lo ininteligible en lo sexual: placer sexual entre personas del mismo sexo.

En esa década, se da un salto cualitativo respecto a los periodos anteriores, se crea un espacio de encuentro de sexualidades masculinas disidentes por medio de la inauguración de la discoteca Oráculos en San Salvador (Gayelsalvador, 2008). La discoteca surge en 1976 con la clara motivación de crear un lugar de encuentro diferente a los existentes en el centro de San Salvador. $\mathrm{Su}$ ubicación se daban en una de las avenidas capitalinas centrales de mejor acceso para personas profesionales que deseaban evitar, probablemente, los riesgos sociales de visitar los puntos de encuentro marginales del centro de San Salvador.

Por su parte, los hombres que ejercitaban el travestismo y que poseían un mejor nivel económico tuvieron en Oráculos una plataforma para manifestar su orientación sexual, sin los riesgos que conllevaba el estar en la calle. Los shows de travestis fueron la táctica para atraer clientes a la discoteca. El uso del travestismo como espectáculo, aunque no escapa del patrón heterosexista binario, sirve en este caso para crear una cápsula de libertad de identidad y paz ficticia en cada noche que la discoteca estaba abierta.

El surgimiento de este espacio de encuentro para personas gais y travestis en San Salvador se puede enmarcar en el fenómeno social de proliferación de grupos antes del comienzo de la guerra. Desde el análisis de luchas de clase propuesto por Martín-Baró (2008: 211): «esa proliferación de grupos era la prueba de que la creciente toma de conciencia de diversos sectores sociales sobre sus intereses de clase buscaba su canalización organizativa en unidades grupales dinámicas». Para el caso de Oráculos y el sentimiento de pertenencia que se constituyó en este periodo, está muy alejada de los planteamientos de intereses de clases; más bien se procuraba un espacio que brindara la libertad de expresar la orientación sexual de sus clientes y propietario, constituyéndose como un proceso de resiliencia para soportar el ambiente hostil de la represión política previo al inicio de la guerra interna que toda la población sufría y la liberación 
del peso asfixiante de las normas homofóbicas de la cultura salvadoreña, aunque sólo fuera por unas cuantas horas.

Oráculos fue para muchos de sus clientes el espacio para vivir su orientación sexual, proporcionándoles un sentido de vida inmediato, ya que la posibilidad de muerte era tan constante que las perspectivas de futuro eran realidades oníricas de una utopía quimérica.

\section{4. ¿Una guerra de sólo heterosexuales?}

Al inicio de la década de 1980 las condiciones sociales y políticas en El Salvador promueven el episodio más oscuro de su historia contemporánea reciente: la guerra. Tuvo una duración de 12 años, de 1980 hasta 1992 y generó un saldo de 75.000 muertes de personas no relacionadas a los bandos en contienda y la diáspora salvadoreña del $20 \%$ de la población en ese entonces (Sue-Montgomery y Wade, 2006: 129). Los 12 años de guerra interna fueron un desgaste a todos los niveles de la vida cotidiana en El Salvador.

Como hemos de suponer, en el contexto machista y heterosexista-normativo, la guerra es la máxima oportunidad para mostrar quien es un hombre de verdad. En este caso, es desconocida la existencia de personas homosexuales al interior de la guerra de forma explícita. El único referente de modificación del patrón esencialista de los roles de género en la guerra fue la participación de la mujer en las líneas del Frente Farabundo Martí para la Liberación Nacional (FMLN). Participación que no está exenta de contradicciones, ya que para ser parte de un proceso tan masculinizado como lo es la guerra, las mujeres tuvieron que realizar un doble esfuerzo que consistió en oprimir lo femenino en ellas por la causa y adquirir comportamientos machistas que demostraban que estaban aptas para participar en la guerra (Vázquez, Ibánez y Murguialday, 1996).

Para encontrar algunas pistas sobre las expresiones de sexualidades masculinas disidentes en la guerra, recurro a la Literatura Testimonial. ${ }^{6}$ En ella existen algunas referencias puntuales expresas e implícitas a prácticas sexuales disidentes. Para empezar, y continuando con la gramática cultural de la violencia en el ejercicio de la sexualidad como instrumento de poder, nos encontra-

6. Género literario que emerge para narrar la realidad latinoamericana al interior de las dictaduras militares y de la represión política y social que se manifestó en diferentes formas de violencias. En él se unen la literatura y la historia con una perspectiva política que rescata la voz de los marginados para evitar su olvido, narrando su propia historia y como tal accediendo a la memoria. La literatura de testimonio se constituye en un contrapeso al monólogo del discurso oficial de la historia. 
mos con una clara referencia de esta situación en la obra Un día en la vida, en la que los efectivos del ejército de la Fuerza Armada hacen una representación simbólica de la identidad sexual masculina esencial exacerbada, la cual podía disponer de los cuerpos y la vida de cualquiera que renegara su autoridad. Pero esa es otra historia para contar en otro momento.

Un día se atrevieron a lo peor. Algo que nos hizo morir: el padre Luna fue encontrado medio muerto en el camino hacia Kilómetro. Le habían dejado la cara desfigurada, con heridas por todas partes. Alguien pasó por el lugar y vio el hombre desnudo que se lamentaba debajo de un barranco. Le habían metido un palo en el ano y todavía lo tenía allí (Argueta, 2005: 38).

Pasando de la literatura a los hechos que le dieron vida, se tiene conocimiento de que un $76 \%$ de prisioneros políticos hombres sobrevivientes a los cuerpos de represión, reportaron al menos una forma de tortura sexual en el periodo de la guerra (Stemple, 2009), y según el informe de la Comisión de la Verdad, la violencia sexual antecedía a la muerte de los prisioneros (Organización de las Naciones Unidas, 1993). La liturgia de la violencia debía realizar un acto de violencia sexual antes de la eliminación de una persona, ya que «sin la sumisión de los cuerpos por la sexualidad, la soberanía política quedaría truncada» (Lara-Martínez, 2012: 32). O tal vez, las violaciones masculinas ejercidas por parte del ejército son un posiblemente desahogo de una identidad sexual proscrita sin el riesgo de ser degradado al campo de lo abyecto para quien realizaba la penetración.

Por su parte, en Las mil y una historias de Radio Venceremos, que narra las experiencias vividas por el equipo de producción de la radio en la zona de guerra en el departamento de Morazán, el carácter de travesti del enemigo utilizado por la izquierda vuelve a hacerse presente, ahora como táctica militar. En este caso los militares, representantes políticos y personalidades de la burguesía, son representados como travestis u homosexuales para desestabilizarlos a nivel personal y social; cuando se trataba de oficiales, se agrega el aspecto militar, tal como expresa el siguiente extracto a nivel de ejemplo:

Al entonces jefe del ejército, el general Vides Casanova, le pegamos lo de Tuti Fruti. ${ }^{7}$ Este general era uno de los mejores estrategas de las filas del enemigo. Es uno de los escasísimos militares que ha entendido la dinámica de la guerra moderna, su componente político. Pero lo de Tuti Fruti le descontrolaba la vida. Luego fue peor, desde que apareció Lotario, un negrón que lo mataba de

7. Ensalada preparada con diferentes frutas. 
celos y que se lo peleaban entre Tuti Fruti y otro oficial. Fíjate qué interesante, Vides Casanova no es maricón. El sí es un tipo todo educado, elegante, de ojos verdes, y para colmo se puso Esmeralda como pseudónimo. O sea, que tenía todos los ingredientes para tildarlo de culero. Y así lo sacábamos una y otra vez en la "Guacamaya subversiva», ${ }^{8}$ como culero. Y de tanto machacar, hasta los mismos soldaditos se lo acabaron creyendo y miraban con desconfianza a su superior (López Vigil, 2006: 429).

Cabe en este punto resaltar las palabras «Vides Casanova no es maricón», relacionando esta afirmación al nivel de educación, elegancia y el aspecto físico para determinar que no era un travesti pobre que frecuentaba la zona de la Praviana para prostituirse.

Este posicionamiento discursivo nos deja entrever que aquella persona sin nivel educativo, que no tiene elegancia (la cual muchas veces es relacionada con las prendas de vestir) y con una apariencia física que se aleje del hombre caucásico cualquiera puede ser designada como culero, loca, maricón, pipián o, de las 100 formas más que existen en el léxico popular salvadoreño para referirse a hombres que se supone que son homosexuales (Romero, 2009: 72-75).

No obstante, en Las mil y una historias de Radio Venceremos se encuentra una de las primeras referencias explícitas a la vivencia de sexualidades masculinas disidentes.

También se respeta la homosexualidad. Mirá a Nando el sastre, que le ha hecho los uniformes a media BRAZ. ${ }^{9}$ Nando pedía permiso como todos y se iba a gatear con su amigo. Y nadie le reprochaba que fuera maricón (el único problemita con Nando es cuando te quiere medir el tiro del pantalón con la mano... jun momento!). Aquí estaba uno que se quiso llamar Lucha Villa. Venían los cuilios y él se daba riata ${ }^{10}$ como todos. Había lesbianas. ¿Quién no supo de los amores turbulentos entre Trini y no me acuerdo la otra? ¿Y qué? Lo del pluralismo vale también para los corazones (López Vigil, 2006: 488).

Este testimonio da cuenta de la utilización de los conceptos de homosexualidad y lesbianas; por el contrario, la identidad trans no posee textualidad en esta época. Ahora bien, la palabra maricón, que en forma general tiene un uso peyorativo en la cultura hispana, se utiliza previo al comentario explicativo de una posible transgresión en lo sexual a un hombre al que se cree heterosexual

8. Nombre de uno de los programas de radio producidos por Radio Venceremos.

9. Brigada Rafael Arce Zablah.

10. Expresión para luchar, golpear y/o pelear. 
y con dicha acción sexual cuestionaría su estatus hetero-normativo. El uso del adjetivo cuilios, derivación del cuiloni anteriormente descrito, es una calificación para degradar al campo de lo afeminado/travestido a los efectivos de la fuerza armada por parte de los guerrilleros, los cuales con este acto lingüístico asumen una posición de jerarquía superior respecto a los otros, aunque sin llegar a la violencia sexual física. El informe de la Comisión de la Verdad para El Salvador señala que es notable la inexistencia de casos de violencia sexual que se le imputa al FMLN (Organización de las Naciones Unidas, 1993). Esta situación se puede comprender, que al designar como cuilios, travestis y homosexuales a los oficiales del ejército y a los soldados, realizaban un acto simbólico de violencia sexual que exaltaba su masculinidad hegemónica reafirmando la homofobia, sin necesidad del sometimiento del cuerpo físico del oponente.

«Lo de pluralismo vale también para los corazones». Aunque esta frase de forma general da una sensación de inclusión, estamos muy alejados de la realidad. En las líneas del FMLN existía una homofobia explícita. Al fundamentar sus acciones políticas dentro de las corrientes ideológicas del marxismo, leninismo, guevarismo y comunismo, siguiendo la línea argumentativa de Wittig (2010: 40), el FMLN asume de manera implícita y naturalizada que todos aquellos fenómenos que salieran del marco doctrinario de la lucha de clases, en este caso la homosexualidad, son considerados como problemáticas burguesas que tendrían fin con el triunfo de la revolución y la dictadura del proletariado. Sin embargo, y coherentes con el principio fundamental de la entrega a la lucha revolucionaria, si el homosexual guerrillero demostraba valor y compromiso suficiente y reprimía sus comportamientos en público, podía encontrar cabida en el FMLN (Vázquez, Ibánez y Murguialday, 1996: 187).

Lo anterior estaba aconteciendo en las zonas de guerra en El Salvador, pero al interior de la capital existía otra dinámica, basada en los diferentes ingresos económicos que cada persona homosexual poseía a nivel individual. En este caso las travestis pobres están confinadas a sitios marginales y excluidos para poder expresar su identidad de género, recurriendo al ejercicio de la prostitución de calle como medio de sobrevivencia.

En el contexto de continua violación de los Derechos Humanos durante la guerra, esta población excluida no escapó de los cuerpos represivos. ${ }^{11}$ Los miembros del ejército llegaban a la zona de la Praviana y, con presión y amenazas, ejercían violaciones sexuales a las travestis, además de exigirles sumas

11. Información proporcionada por Joaquín Cáceres en una entrevista realizada el 20 de agosto de 2014. 
de dinero que rondaban los 50 dólares, cifra exorbitante para esa época. Las que no aceptaban pagar esa cantidad eran sometidas a otros tipos de violencia y agresiones a sus identidades. Por ejemplo, a algunas les cortaban el cabello y a otras las llevaban a la base militar, donde eran sometidas a un proceso de violación colectiva por parte de los soldados. No en pocas ocasiones, varias de estas travestis desaparecieron y otras fueron localizadas muertas en la zona conocida como el Playón. En este continuo de agresiones, en el año $1984,{ }^{12}$ se conoce un caso paradigmático de la muerte un grupo de 12 travestis en la segunda Avenida Norte, las cuales fueron brutalmente violadas y asesinadas. Existió una denuncia ante la Comisión de Derechos Humanos, pero esta fue archivada y olvidada.

En la época de la guerra interna existieron menos espacios para discutir las manifestaciones disidentes de la sexualidad que en la época anterior. En el ejército, realizar esta discusión era imposible, por ello se efectuaba un desahogo de estas identidades proscritas por medio de violaciones que incluían a hombres y travestis. En la burguesía cafetalera, la cual tenía la facilidad de movilizarse fuera del país, ${ }^{13}$ algunos de sus integrantes deben haber ejercicio esas sexualidades disidentes en espacios anónimos de la gay night life en Estados Unidos. También los hijos de la burguesía cafetalera eran enviados a estudiar a los Estados Unidos, Canadá, México o países de Europa, donde las normas homofóbicas de El Salvador podían tener flexibilidad respecto a prácticas sexuales con otros hombres. En 1984 se reportó el primer caso de VIH en etapa avanzada en un hombre homosexual de 33 años, que adquirió la enfermedad en los Estados Unidos (El Diario de Hoy, 2006). Este hecho reforzó los patrones homofóbicos en la sociedad, más aún considerando el desconocimiento que se tenía sobre esta enfermedad.

Por su parte, en la filas del FMLN, los temas sobre sexualidad no entraban en las discusiones políticas; si una persona lesbiana u homosexual daba muestras públicas de estas tendencias, era algo anormal que no podía estar ahí, imposi-

12. Fecha histórica que la Asociación Entre Amigos maneja sobre este evento. No obstante, Lester y Chávez (2016) cuestionan esta fecha, ya que en primer lugar no está contrastada y, en segundo lugar, tras realizar indagaciones con un sobreviviente de ese evento, se recuerda una redada policial en las proximidades del monumento a El Salvador del Mundo, donde desaparecieron como mínimo 12 mujeres trans. Después de procurar información en noticias de periódicos, estos autores proponen octubre de 1980 como fecha probable de ejecución de este acto de masacre de mujeres trans en El Salvador.

13. Ir a Nueva York, San Francisco y Miami desde El Salvador son entre tres y cinco horas de vuelo. 
bilitado en ocasiones a homosexuales el ascenso a cargos de dirección al interior de la estructura del FMLN (Vázquez, Ibánez y Murguialday, 1996: 271).

La participación de las mujeres y sus vivencias en las líneas de guerra mostró que los esencialismos sexuales pueden ser susceptibles de transformación, pero cuando miramos hacia «[...] el prejuicio homo y lesbofóbico, sin embargo, no fue alterado. Las preferencias sexuales distintas a la heterosexualidad eran toleradas siempre y cuando no perturbaran la entrega a la causa y se mantuvieran en silencio» (ibíd: 189).

La discoteca Oráculos continúa abierta y desarrolla actividades para la Comunidad Gay Salvadoreña (CGS) (Saade, 1989 b), nombre con el cual se pueden identificar las personas que frecuentan la discoteca, tanto clientes como los encargados de presentarse en los espectáculos de travestis. La CGS de Oráculos tampoco escapaba a las actividades de represión en la época de la guerra. Así, Julio Saade, propietario de Oráculos, narra que en julio de 1989 sufrieron un allanamiento por parte de cuerpos de seguridad, al igual que otros centros nocturnos de la capital. La razón aparente era atrapar personas nones gratas antes de las fiestas patronales de agosto de la capital (1989 a). La temática sobre el VIH también estaba presente al interior de Oráculos (Saade, $1988 a, 1988 b$ ), tanto es así que posiblemente los efectos negativos de la epidemia motivaron los lemas de identificación de la discoteca en los últimos años de la década de 1980: «No drogas, no armas, no sexo» (Saade, 1989 a) $\mathrm{y}$ «No a las drogas, practica el sexo seguro» (Saade, 1989 b). Debido al VIH, se documenta una de las primeras acciones gubernamentales para dar atención a la población homosexual cuando la Comisión Nacional contra el Sida (Conasida) patrocinó el evento de Miss Gay 89/90 (Saade, 1989 b). También existió interés por reivindicar sus derechos humanos ante la sociedad (Saade, $1989 a$ ).

En 1988 se produce el primer trabajo de grado que relaciona el VIH y la homosexualidad como parte de la carrera de piscología de la Universidad Francisco Gavidia (Villatoro y Quintero, 1988). Como primer elemento de interés se encuentra el título de esta obra, ya que la homosexualidad no se manifiesta como orientación sexual sino como práctica sexual. En este estudio se contactaron 120 sujetos pero al final solo participaron 100 voluntarios con «características homosexuales». Prácticamente fueron adolescentes y jóvenes los que participaron en este estudio (ibíd.: 56), muestra de ello es que el $40 \%$ del total fueron estudiantes universitarios, aunque también existieron profesionales universitarios y no universitarios, estudiantes de bachillerato y de educación básica, además de un $24 \%$ que no especificó su nivel académico. Otro aspecto relevante de este estudio es la autopercepción de aceptación o rechazo que este 
grupo posee respecto a la homosexualidad en El Salvador de 1987. Así, el mayor porcentaje de aceptación fueron los amigos, con un $86 \%$, y el mayor rechazo se encuentra en la sociedad en general, con el $39 \%$ (Ibíd.: 53). Entre los lugares donde se desarrollaron las entrevistas encontramos la discoteca Oráculos, aunque no se nombra como tal en este trabajo.

\section{Conclusiones}

En los sesenta años de historia de las sexualidades masculinas disidentes analizadas nos hemos encontrado con un patrón de la homofobia que conduce al menosprecio, marginación, exclusión hasta la tortura, desapariciones y asesinatos. Esta homofobia se fundamenta en la promoción de la ignorancia, el silencio de la sexualidad y la ocultación del cuerpo, lo cual se convierte en dispositivos para perpetuar la masculinidad hegemónica heteronormativa sexista en El Salvador.

La jerarquía sexual, fundamentada en el binomio opresor-oprimido, marca las relaciones de poder en las relaciones de género, donde todo lo relacionado a lo femenino es el objeto y sujeto de opresión. Cuando hablamos de sexualidades masculinas disidentes, las cuales se remiten a lo femenino, la masculinidad hegemónica lo interpreta como una alteración que desestabiliza el orden binario sexual y de opresión. Por eso, quien ejerza ese tipo de sexualidades, según la epistemología de la violencia construida, debe ser eliminado. Esta eliminación ha adquirido dos formas de expresión: la física y la social. En el juego de polarización izquierda y derecha al interior de El Salvador hemos visto en las páginas anteriores su proceder: la violación, tortura, desapariciones y asesinatos los ejecutan sectores más conservadores; mientras que la izquierda recurre a procesos simbólicos de muerte social por medio de la feminización del oponente político y/o militar.

La vivencia y el ejercicio de sexualidades masculinas disidentes transitaron entre lo marginal y lo privado en esta época histórica analizada. Vimos que el centro histórico de San Salvador, con sus bares y antros, era un lugar propicio para establecer encuentros sexuales, y con el surgimiento de la discoteca Oráculos se crea un primer espacio disidente reconocido que transitó desde lo marginal y privado hasta un cierto espacio público restringido de sociabilidad travesti. Los hombres que ejercían sexualidades disidentes se exponían a mayores o menores riesgos, según fueran sus condiciones económicas. 
La politización de las sexualidades masculinas disidentes en este periodo histórico no aconteció. Esta eclosión tendría que esperar hasta después de 1992, y todavía 17 años más para establecerse como un actor de la agenda política nacional, gastando sus energías en deconstruir la imagen de marginación, exclusión y opresión con que el imaginario cultural salvadoreño designa a todo aquello que escape de la norma binaria heterosexual. Pero esa es otra historia para contar en otro momento.

\section{Referencias bibliográficas}

Argueta, M. (2005): Un día en la vida, San Salvador, Dirección de Publicaciones e Impresos.

Audiovisuales de la UCA (2007): 1932, la negación indígena [documental], San Salvador, UCA.

Barrientos Delgado, J. (2015): Violencia homofóbica en América Latina y Chile, Santiago de Chile, Ediciones y Publicaciones El Buen Aire.

Bourdieu, P. (1999): A dominação masculina, Rio de Janeiro, Brasil.

Butler, J. (2005): Cuerpos que importan: sobre los límites materiales y discursivos del «sexo», Buenos Aires, Paidós.

- (2007): El género en disputa. El feminismo y la subversión de la identidad, L'Hospitalet de Llobregat, Paidós.

- (2010): Marcos de guerra. Las vidas lloradas, Buenos Aires, Paidós.

El Diario de Hoy (2006): Sida cumple 25 años, 6 de junio de 2006.

Foucault, M. (1972): A arqueologia do saber, Petrópolis, Editora Vozes.

Gayelsavador.com (2008): «La historia de Oráculos», Portal gaysalvador. com, recuperado de: www.gayelsalvador.com/oráculos.htm. Fecha de acceso: 21 de noviembre de 2014.

GRÉGORI, R. (2007): «Amor homosexual y lésbico en el arte salvadoreño», $E l$ Faro, Recuperado de:http://archivo.elfaro.net/secciones/el_agora/20070709/ ElAgora1_20070709.asp. Fecha de acceso: 3 de abril de 2014.

LARA-MARTínez. R. (2012): Indígena, cuerpo y sexualidad en la literatura salvadoreña, San Salvador, Editorial UBD.

Lester, J.; N. Chávez (2016): «Lo que les pasó a las mujeres trans que desaparecieron en El Salvador», Buzz Feed News World, recuperado de: http://www.buzzfeed.com/lesterfeder/lo-que-les-paso-a-las-mujeres-transque-desaparecieron-en-el\#.10411eV7n. Fecha de acceso: 21 de enero de 2016. 
López Vigit, J. (2006): Las mil y una historias de Radio Venceremos, San Salvador, UCA Editores.

MARTín-BARó, I. (2012): Acción e ideología: psicología social desde Centroamérica, San Salvador, UCA Editores.

- (2008): Sistema, grupo y poder: psicología social desde Centroamérica (II), San Salvador, UCA Editores.

Martínez, O. (2004): El Salvador, del conflicto armado a la negociación 1979-1989, San Salvador, Editorial Nuevo Enfoque.

Prudhmme, O. (2010): «Historias pioneras, fuentes inéditas. Del carácter pionero de las tesis de licenciatura en Historia escritas en El Salvador», Identidades, 1: 92-103.

Organización de las Naciones Unidas (1993): Informe de la Comisión de la Verdad. De la locura a la esperanza. La guerra de 12 años en El Salvador, Nueva York, Naciones Unidas.

Organización Mundial de la Salud (2014): Global status reporto violence prevention, Luxembourg, WHO.

Ramírez, S. (1985): Salarrué: el ángel del espejo, Barcelona, Bodoni.

Real ACademia Española (2006): Diccionario Esencial de la Lengua Española, Pozuelo de Alarcón.

Romero, M. (2009): Léxico del Cuerpo Humano en el habla popular salvadoreña, San Salvador, Editorial Delgado.

SAAde, J. (1988 a): Nuevo Mundo, folleto informativo, mes de junio, San Salvador, Discoteca Oráculos.

- (1988 b): Nuevo Mundo, Folleto informativo, mes de julio, San Salvador, Discoteca Oráculos.

- (1989 a): Nuevo Mundo, Folleto informativo, mes de julio, San Salvador, Discoteca Oráculos.

- (1989 b): Nuevo Mundo, Folleto informativo, mes de septiembre, San Salvador, Discoteca Oráculos.

Salazar Arrué, S. (2010): Narrativa Completa II, San Salvador, Dirección de Publicaciones e Impresos.

Stemple, L. (2009): «Male Rape and Human Rights», Hastings Law Journal, 60: 605-647.

Sue-Montgomery, T.; C. WAde (2006): A revolução salvadorenha: da revolução à reforma, São Paulo, Editora da UNESP.

Ernesto «San AviléS» (1974): San Sebastián [Pintura], Madrid.

Vázquez, N.; C. Ibánez; C. Murguialday (1996): Mujeres Montaña. Vivencias de guerrilleras y colaboradoras del FMLN, San Cristóbal, Horas y Horas. 
Villatoro, M.; R. Qintero (1988): El sida y su incidencia en el cambio de actitudes hacia la práctica homosexual de la población masculina en la ciudad de San Salvador, trabajo de grado en Psicología, San Salvador, Universidad Francisco Gavidia.

Wittig, M. (2010): El Pensamiento Heterosexual y otros ensayos, Móstoles, Top Printer Plus. 